\title{
FROM UNDERSTANDING TO ACTION: AN EXAMINATION OF TRANSFORMATIVE LEARNING IN ASYNCHRONOUS ONLINE EQUITY, DIVERSITY, AND INCLUSION TRAINING FOR FIRST- TIME TEACHING ASSISTANTS
}

\author{
Cori Hanson and Mikhail Burke \\ University of Toronto \\ cori.hanson@utoronto.ca and mikhail.burke@mail.utoronto.ca
}

\begin{abstract}
To better support TAs in creating inclusive classrooms, three (3) online, asynchronous modules were developed and implemented to introduce first-time TAs to core concepts of equity, diversity, and inclusion (EDI) Foundational EDI Language, Power, Privilege, and Positionality and Interrupting Bias. Over 100 1st time TA completed each module, with 80-90 providing feedback on their experience upon completion. A preliminary review of this feedback highlighted three major themes: 1) building awareness and knowledge, 2) applying EDI concepts to teaching practice \& identifying actions, and 3) feeling empowered to act. Overall, TAs expressed strong development of awareness and new knowledge of key concepts such as equity and positionality. Although TAs were also able to identify and state the value of applying these concepts to their teaching practice, many expressed the sentiment of still feeling uncomfortable to act within "real-life" situations. Future iterations of such training could seek to address this through structured opportunities for analysis and feedback of reflective responses.
\end{abstract}

Keywords: equity, diversity and inclusion training, TA training, transformative learning, asynchronous online learning

\section{INTRODUCTION}

Engineering education, both in content and practice, is a socio-technical process. Therefore, in developing prospective practitioners, such as first-time Teaching Assistants (TAs), in the space supplying opportunity to develop social aspects of instruction including inclusive pedagogy and practices is just as vital as building technical proficiency. Being able to create an inclusive learning environment is essential for TAs as they are often teaching in smaller classes providing the opportunity to build community, trust and connections with their students not always found in large lectures.
A foundation for creating an inclusive learning environment is an understanding of positionality and power dynamics in the classroom yet TAs are not often provided the opportunity to develop the skills necessary to do this work. Equity, diversity, and inclusion (EDI) was introduced to our Faculty-wide TA training in 2018 with the purposed of training TAs to be able to create inclusive classrooms. This training was originally held in-person and provided a brief overview of key EDI concepts and definitions, the reasoning behind why the Faculty believed EDI was important in teaching, as well as opportunities for reflection and practice through case studies and discussion with peers and the facilitators.

In 2020, this training was moved online due to COVID19 and was adapted into three asynchronous online modules with the same purpose of introducing first-time TAs to the core concepts of EDI and help them develop practices to create inclusive classrooms.

Generally, EDI training introduces participants to concepts related to equity, diversity, and inclusivity issues with the intention of building awareness and promoting changes for more equitable and inclusive practices. For example, professional organizations may provide bias training so that members or employees have a better understanding of their biases and how to mitigate them. An example of this is the Toronto Initiative for Diversity \& Excellence (TIDE) at the University of Toronto, which offers unconscious bias education to search committees or other decision-making bodies with the intention of helping mitigating biases which could be a barrier for diversity [1]. This practice aligns with transformative learning which proposes that when a learner critically reflects on new knowledge that is different than their existing beliefs this can result in new beliefs and behaviours [2, 3]. This was also the overarching goal of the Faculty-wide EDI TA training modules; the training was developed to provide new TAs the opportunity to reflect on EDI concepts and issues in the context of their teaching and role as TAs to 
inform their practice as educators and support the creating of more inclusive classroom communities.

At our Faculty TA training is provided before the start of the term, at the end of August or early September. This is to ensure new TAs have the pertinent support and the information they need before they enter the classroom. The new online EDI TA training modules were developed to be asynchronous for accessibility. This modality meant TAs would have the same learning experience regardless of their time zone, work schedule or living situation. We recognized that not all TAs would be able to attend a synchronous session and the resources were not available to host multiple sessions at this time of year. We also did not feel that a recorded version of a synchronous workshop would provide the same learning experience for TAs who could not attend the session live. Further, asynchronous modules would allow TAs to work at their own pace, perhaps providing more time to reflect on what they were learning, something not always possible in a synchronous workshop.

Developing the training to be asynchronous offered an exciting opportunity for innovation in EDI education. However, since asynchronous EDI training is not common and the literature on transformative learning in asynchronous online education is limited, it was somewhat unknown whether this training would effectively facilitate first-time TAs applying new knowledge to transform their developing teaching practices.

\subsection{Purpose}

This paper summarizes our initial findings from a preliminary programmatic review of the new modules and whether transformative learning is a potential outcome of asynchronous online EDI training for early-career engineering educators. This work was guided by the following questions:

1) Was asynchronous online learning effective for EDI training? What worked and what did not?

2) Is there potential for transformative learning in asynchronous online learning modalities?

\section{BACKGROUND AND FRAMEWORK}

\subsection{EDI Training in Higher Education}

Over the past several years, there has been an increased desire for the explicit integration of EDI considerations and values within the operations of various industries and institutions including higher education [4] and academic research [5]. With many individuals often expressing a lack of knowledge or understanding about concepts within the EDI space and how it may intersect with their profession, a common tactic is to create opportunities to leverage EDI training or resources to build individual capacity for action with the desired outcome being increased equity, diversity and/or inclusion. The focus of such developed EDI training/resources can typically be defined into two major buckets: building capacity for EDI integration within content/deliverables and building capacity for EDI integration within environmental culture and practice.

For spaces such as engineering Faculties, building capacity for EDI integration within both content and culture is key in both engineering pedagogy and research. This is being reinforced through organizations such as the Canadian Engineering Accreditation Board (CEAB) reinforcing assessment of ethics and equity for required graduate attributes [6] as well as Tri-Agency organizations (such as the Natural Sciences and Engineering Research Council of Canada - NSERC) calls for more explicit EDI consideration within grant proposal regarding research methods and development of lab personnel [7]. This means institutions are motivated to empower instructors to build more direct links between EDI principles and curricular content, to empower researchers to integrate an EDI lens in scoping research questions and approaches, and generally to create more inclusive spaces.

While a variety of developed resources exist that instructors and teaching assistants could leverage in building EDI capacity and integrating tool/ideas into practice $[7,8,9,10]$, self-instruction can often act as barrier for individuals who do not feel empowered to learn the content or are unsure where to begin especially when it is felt that EDI initiatives and work does not fully align with institutional culture or professional identity as is sometimes the case in the context of science, technology, engineering and mathematic disciplines [11, 12]. Structured online training, whether synchronous or asynchronous, guides participants through content and scaffold relevant resources and tools.

EDI training/workshops can focus on a variety of different topics which can then be framed within the operational context and common positionality of the participants as exampled by the Anti-Racism and Cultural Diversity Office of the University of Toronto [13]. While such EDI training and opportunity engagement is often optional in nature, due to the requirements of external bodies such as CEAB and the Tri-Agencies, there is growing calls for such training to be mandatory in nature. Universities such as the University of Alberta are currently leveraging working groups to develop EDI training materials for mandatory training for its faculty and staff [14]. While there is debate on who can be mandated to take what training within academic institutions, for stakeholder groups such as TAs who already have mandatory training, integrating EDI training into an existing infrastructure makes such training more accessible for participants who are developing their pedagogical skillsets.

To our knowledge most EDI training is held synchronously and usually in person. None of the EDI initiatives we found utilized asynchronous modules to do this training. 


\subsection{Transformative Online Teacher Education}

Mezirow's transformative learning theory [2, 3] describes how a learner's perspective can change through the learning process to result in new beliefs, behaviours, and actions. Mezirow describes this as

"the process of becoming critically aware of how and why our presuppositions have come to constrain the way we perceive, understand, and feel about our world; of reformulating these assumptions to permit a more inclusive, discriminating, permeable, and integrative perspective; and of making decisions or otherwise acting on these new understandings" [2, p. 14].

In adults, transformative learning occurs when a learners existing beliefs are challenged by new knowledge followed by periods of critical reflection and opportunities for further discourse on the learning [3]. Learners leave a transformative learning process having changed not only their own learning schemes but often their views of themselves and others have also changed.

Online teacher education and professional development provides a medium for flexible and personalized training that allows for the development of knowledge and applied skills as well as access to a community of practice that could better meet the needs of adult learners [15]. Dialogue and opportunities for discussion around content and course related topics are crucial for online learning [16] and effective online teacher education [15, 17]. Further, transformative learning has been observed in teachers who have actively engaged in online discussions especially when there was dialogue between teachers with different points of view [17]. The literature indicated transformative learning in teacher education was possible and achievable online, however, similar to EDI training we were unable to find any studies that looked at transformative learning online teacher education that utilized only asynchronous modules.

\subsection{Overview of the Asynchronous Online EDI Training Modules for First-time TAs}

The three EDI modules were developed with the understanding that the group of first-time TAs would have varying levels of EDI knowledge and competency. To address this, we structured the three modules so that there was a module for beginners i.e., TAs new to EDI, a module that was more intermediate and looked at more complex EDI concepts and finally an advanced module that provided the opportunity to apply EDI knowledge and reflect on future actions.

The beginner module, titled Foundational EDI Language, articulated how language is important in EDI practices. The module was developed to introduce TAs to inclusive and equitable terminology. We described this module as "great for TAs who want to build a strong foundation for equitable and inclusive teaching."
The intermediate module was titled Power, Privilege, and Positionality. This module was meant to provide TAs with the opportunity to learn about and reflect on the power dynamics and interpersonal relationships in the classroom. It was described to TAs as a "great modules for those interested in the theories that ground equitable and inclusive teaching practices."

The advanced module titled Interrupting Bias, was developed to teach TAs how and why biases occur and most importantly how to respond and interrupt biased comments or incidents that could be harmful or problematic in a classroom. This was intended to be a module for TAs who wanted to learn practical ways to maintain an inclusive learning environment.

The learning outcomes for each module can be found in Appendix A. While not assessed in this paper, they provide further context to the objectives of each module and the goals of the facilitators.

Content in the modules was delivered through written text and videos. All three modules employed reflective activities, i.e., self-reflective journaling style prompts and mini-case study analysis, which engaged TAs in the process of applying the content they were learning to various situations inside and outside the classroom. Each module also had a corresponding discussion board where TAs could post questions, discuss module content, and get feedback from their peers and the training facilitators.

At the end of each module TAs were asked to complete a short feedback survey that was embedded into the module on the learning management system. The feedback survey for all three modules was the same and consisted of three open-ended questions: 1) What were your key takeaways from this module? What did you learn? 2) What is still unclear after completing this module? What questions do you still have? 3 ) Is there anything else you would like us to know about your experience completing this module? These feedback surveys were optional, but most TAs completed at least some or all the questions. The feedback surveys each had $\sim 80-90$ responses.

All first-time TAs at the Faculty were added to a TA Training course built in our institutions learning management system and were required to complete two hours of asynchronous training through this course. Completing at least one of the three EDI modules was a mandatory part of this training. TAs were not removed from the course after completing the training; TAs had access to all resources and module content after completing the training.

Each module was completed by $80-130$ TAs. The TAs were all going to be teaching for the first-time as a TA in our Faculty in Fall 2020, there was no knowledge of whether they had previous teaching experience or EDI training. The TAs were all engineering students studying at our Faculty. Most of the TAs were graduate students at both the masters and doctoral levels. Masters' students were enrolled in both MEng \& MASC degrees. 


\section{FINDINGS}

This programmatic review of the TA feedback collected by the module feedback surveys found the following themes: 1) building awareness and knowledge, 2) applying EDI to teaching practice \& identifying actions, and 3) feeling empowered to act.

\subsection{TAs Building Awareness and Knowledge of Key EDI Concepts}

Within all three developed modules, there was a defined learning outcome centred around building an understanding for the specific terminology and concepts introduced within the module. In each module, there was typically one concept/idea which stood out more prominently than others. For the Foundational EDI Language module, many TAs expressed how they learned the difference between the idea of equality versus equity in the context of fairness. Considering how the engineering mindset can be associated with positivism and the myth of objectivity [18], there is value in such term delineation facilitating student knowledge growth in what it means to be equitable. For the Power, Privilege, and Positionality module, many TAs expressed new understanding and awareness of how one's life experience and identity could influence (and perhaps bias) their perspective (positionality). Again, this is valuable as various studies have highlighted the importance understanding the role of positionality within both education $[19,20]$ and qualitative research [21].

For the Interrupting Bias module, many TAs expressed an understanding of bias and implicit bias. Several TAs expressed comfort with the acknowledgement within the training module that having biases "doesn't make you a bad person." This is a key takeaway. As shown in other work [22], highlighting one's implicit biases through tools such as the Implicit Association Test (which was also leveraged within the module) can make one be more defensive which in turn could reduce their likelihood of engaging in interrupting bias. Therefore, by clearly addressing the intrinsic biological nature of implicit associations and shifting the conversation from prevention to interruption, the module was able to reduce potential expressions of defensiveness.

\subsection{Applying EDI Knowledge to Teaching Practice \& Identifying a Need for Action}

In addition to disseminating knowledge and content, the EDI modules strived to support the further application of knowledge learned by TAs to their developing teaching practices. Despite having the opportunity to reflect on specific teaching scenarios and practices few TAs articulated an application of this knowledge in their feedback surveys. In the feedback for all three modules, TAs replied that they were still uncertain and/or had questions about how to apply the knowledge they gained to their role as a TAs.

For the Foundational EDI Language and Power, Privilege and Positionality modules this finding was somewhat expected as these modules were more theoretical. TAs wanted to know how to apply what they learned about power and positionality in the classroom specifically and how this applied to their role as a TA. There were prompts for TAs to reflect on their privilege and power during the module. One of these reflections specifically asked TAs to reflect on their power as a TA. In the feedback survey responses on key takeaways from the Power, Privilege and Positionality module, we found that TAs did acknowledge a stronger understanding of their own privilege in the feedback, however, few TAs, articulated this in connection to their role as teachers even though they had been prompted to do so previously in the module. Some did and was seen in comments such as "As a TA, I will need to make use of my power to try to minimize the impact from the privilege and try to resolve conflicts brought by different positionalities."

Another application that was observed in the feedback was in the TA responses on the concept of silence. In the Interrupting Bias module TAs were introduced to the idea that when we are silent this can be interpreted as acceptance and/or agreement. The training utilizes the phrase "What you permit, you promote" to summarize this concept. A key learning mentioned in the TA feedback was the understanding that as a TA it was their responsibility to speak-up and use their power to address harmful bias in the classroom. This was seen in comments such as "The importance, especially as a TA, to ensure the classroom is inclusive. "What you are silent about you allow to continue" and "The main takeaway was that being silent to an issue is being complacent. It is important to voice when something wrong is occurring." So, while TA feedback indicated that they were not always confident in how to act, it would appear that potentially TAs understood that that action was needed.

\subsection{Feeling Empowered to Act \& Interrupting Bias}

A desired outcome of the EDI TA Training is for new TAs to feel empowered and ready to implement more inclusive teaching practices and this includes the ability to address difficult situations such as harmful biases in the classroom. This was the focus of the Interrupting Bias module. This module included a section with suggested phrases that TAs could use to respond to biases as well as multiple mini-case studies based on real scenarios that TAs were asked to reflect on how they would respond in each situation. Some TAs did acknowledge they had no further questions and a few wrote that they understood how to interrupt bias. However, most TAs expressed uncertainty about how to address bias in the classroom and/or asked for more examples of how to do this in practice. In the 
feedback, one TA described how they were not uncertain about any of the content in the module, but they were anxious about having to do this in real life,

"Not unclear, but the tools to respond are entirely so much harder in real life than written down. As a typically quiet (in almost all day-to-day situations), nonassertive/aggressive, and very emotional person interrupting bias can be emotionally challenging for me (never mind the anxiety about whether I have interrupted it in the right way) and reading about it online will not suddenly make me more confident in doing it, even though I know how important it is, and that it has to be done."

This comment brings to attention a limitation of EDI training generally, where learners might be able to intellectualize concepts and understand what they should do in theory or during the workshop but that taking these actions in real-life is often more difficult.

\subsection{Appreciation - An Unexpected Finding}

We hoped the TAs would value the learning experience, but we did not expect TAs to explicitly express appreciation for the opportunity to do this learning, however, some TAs did. We received comments in the feedback saying the training was interesting, important, and useful. We also received a few comments like this one which specifically articulated appreciation as well as a desire for this training to be more broadly disseminated, "I greatly appreciated this module and believe not only TAs, but all students and staff should complete it too." This want for more people in engineering education to complete EDI training was also a finding in a paper on what women in undergraduate engineering programs think their institutions could do to be more inclusive [23]. This is an important finding because many engineering educators and institutions want to know what they can do to be more equitable and welcoming to underrepresented students, staff and faculty but may be hesitant in acting not knowing how the community will react to EDI initiatives. Our work has found that first-time TAs may appreciate EDI training and would support the implementation of this type training for a wider audience.

\section{DISCUSSION}

\subsection{Transformative Learning in Asynchronous EDI Training}

The findings from this programmatic analysis indicate that transformative learning may be possible in the context of asynchronous EDI training. The modules we developed provided the foundation and instigation needed for transformative learning by effectively introducing TAs to EDI concepts. The feedback indicated that key learnings for many of the TAs included the learning of new concepts or that the module provided additional understanding to their existing EDI and teaching knowledge. Occasionally this was also expressed as discomfort.

This EDI training also provided the needed opportunities for critical reflection required for transformative learning through reflective prompts, activities like the IAT and mini-case studies. As a result of these reflective activities TAs expressed their understanding of positionality and power in the context of their own teaching. Many demonstrated the understanding of why action was needed by them as TAs and have a deeper understanding of the risks of staying silent.

However, many TAs still reported a lack of confidence and understanding of how to take actions based on the knowledge they had gained through the training. This last step is crucial in achieving transformative learning [3, 17]. Only a few TAs expressed confidence by clearly stating they had no uncertainties or questions after completing the training. It is possible then that a few students did experience transformative learning and will put their work and knew knowledge into action. However, this change in perception and awareness is not fully known. A shift in perception is implied but measuring this in practice is more elusive and would require a more in-depth study which could include follow-up interviews or observations of teaching.

\subsection{Lessons Learned and Future Work}

The training modules were generally well received and appreciated. TA feedback highlighted several strengths and areas of improvement for the modules as currently constructed. Whenever video content was leveraged over text to highlight concepts, this was typically appreciated by the TAs. The TAs were also more receptive to engaging/interactive activities such as the Implicit Association Test (IAT). Generally, the Interrupting Bias module was the module that was most well received by participants as it was perceived to be the clearest in terms of providing direct and practical means to address unideal situations within the classroom or within one's own mindset.

Conversely, with the Foundational EDI Language module, while there were many situational prompts for TAs to consider how they may address situations that may occur within the classroom as a means of understanding concepts, many participants expressed that still felt unsure how "best" to handle the situation, expressing the desire for more guidance explicit to the situation. For the Power, Privilege, and Positionality module, while student expressed a desire for more situational learning examples, there was also expressed appreciation for the relative depth in reflection and awareness building relative to the other modules.

A clear limitation was that the asynchronous modality made discussion and discourse more difficult; while discussion boards were available and linked to throughout the training, posting on the discussion boards was not 
required to complete the training and were typically not used by the TAs, highlighting the need to facilitate more discussion board participation and/or establish opportunities for ongoing feedback and dialogue on the module content. In future iterations of the training requiring discussion board participation and/or the implementation of additional synchronous sessions either during and/or after the training could be used to provide the opportunity for TAs to engage in further dialogue and work through any concerns, they may have that could not be addressed through the asynchronous modules alone.

The findings from this preliminary program assessment provided the groundwork and reasoning for a more indepth analysis of the online EDI training with transformative learning as an outcome. In the next phase of this work, a more robust research study will be developed to analyze: 1) the reflection responses from module activities, 2) the feedback from TAs who completed all three modules, and 3) whether identity and background in teaching and/or EDI education impact the possibility for this training to be transformative.

\section{CONCLUSION}

Asynchronous online EDI training has the potential to facilitate transformative learning opportunities for firsttime TAs. Our work highlights how this process can be started by effectively introducing TAs to potentially new, complex EDI issues and contexts which may be different than their existing understandings and viewpoints. Similar, to other studies on online teacher training, we believe a limitation to transformative learning in this modality could be whether learners engage in meaningful dialogues about what they are learning. For transformative learning to be achieved in asynchronous training opportunities for dialogue with peers, feedback from the facilitators during and after the training are likely needed.

\section{Acknowledgements}

Thank you to the teaching assistants who provided their thoughtful and helpful feedback making this analysis possible. Thank you to our Faculty's leadership for valuing EDI training enough to make it a mandatory part of the first-time TA training program.

\section{References}

[1] "Toronto Initiative for Diversity \& Excellence (TIDE)," [Online]. Available: https://www.torontotide.ca/. [Accessed 0503 2021].

[2] J. Mezirow, Transformative dimentions in adult learning, San Francisco: Jossey-Bass, 1991.

[3] J. Mezirow, Ed., Learning as transformation. Critical prerpectices on a theory in progress., San Francisco: Jossey-Bass, 2000.
[4] Universities Canada, "Equity, diversity and inclusion at Canadian universities: Report on the 2019 survey," Universities Canada, 2019.

[5] Canada Research Coordinating Committee, "2019-20 Progress Report: Mobilizing Canadian Research," Government of Canada, 2020.

[6] Canadian Engineering Accreditation Board, "2016 Accreditation Criteria and Procedures," Engineers Canada, 2017.

[7] Natural Sciences and Engineering Research Council, "Guide for Applicants: Considering equity, diversity and inclusion in your application," [Online]. Available: https://www.nserccrsng.gc.ca/_doc/EDI/Guide_for_Applicants_EN.pdf. [Accessed 0503 2021].

[8] European Commission, "Gendered Innovations 2: How inclusive analysis contributes to research and innovation," European Union, 2020.

[9] Hartwell et. al, "Breaking Down Silos: Teaching for Equity, Diversity, and Inclusion Across Disciplines," Humboldt Journal of Social Relations, vol. 39, pp. 149 - 162, 2017.

[10] M. A. Moriarty, "Inclusive Pedagogy: Teaching Methodologies to Reach Diverse Learners in Science Instruction," Equity \& Excellence in Education, pp. 252-265, 2007.

[11] S. A. Allen-Ramdial and A. G. Campbell, "Reimagining the pipeline: Advancing STEM diversity, persistence, and success," BioScience, vol. 64, no. 7, pp. 612-618, 2014.

[12] S. E. Brownell and K. D. Tanner, "Barriers to faculty pedagogical change: Lack of training, time, incentives, and...tensions with professional identity?," CBE-Life Sciences Education, vol. 11, no. 4, pp. 339-346, 2012.

[13] Anti-Racism \& Cultural Diversity Office, "Training \& Workshops," [Online]. Available: https://antiracism.utoronto.ca/request-a-trainingworkshop/. [Accessed 0503 2021].

[14] University of Alberta, "Strategic Plan for Equity, Diversity and Inclusivity," University of Alberta, 2019.

[15] K. P. King, "Identifying successin online teacher education and professional development," The Internet and Higher Education, vol. 5, no. 3, pp. 231246, 2002.

[16] N. R. Boyer, P. A. Maher and S. Kirkman, "Transformative learning in online settings: The use of self-direction, metagognition and collaborative learning," Journal of Transformative Education, vol. 4, no. 4, pp. 335-361, 2006. 
[17] K. Lee and C. Brett, "Dialogic understanding of teachers' online transformative learning: A qualitative case study of teacher discussions in a graduate-level online course," Teaching and Teacher Education, vol. 46, pp. 72-83, 2015.

[18] D. Riley, Engineering and Social Justice, 2008.

[19] E. Taylor, E. J. Tisdell and M. S. Hanley, "The Role of Positionality in Teaching for Critical Consciousness: Implications for Adult Education," in Adult Education Research Conference , 2000.

[20] Straton et. al, "Positionality in Teaching: Implications for Advancing Social Justice," The Journal of General Education, vol. 67, pp. 109-121, 2018.

[21] D. Jacobson and N. Mustafa, "Social Identity Map: A Reflexivity Tool for Practicing Explicit Positionality in Critical Qualitative Research," Internation Journal of Qualitative Methods, vol. 18, 2019.

[22] Howell et. al, "Defensive Responding to IAT Feedback," Social Cognition, pp. 520-562, 2017.

[23] C. Hanson, "What women students want their insitutions to do to make engineering education more inclusive and less "chilly"," Proceedings of the Canadian Engineering Education Association (CEEA) Conference, pp. 1-8, 2020.

\section{Appendix A: Training Module LEARNING OUTCOMES}

$\underline{\text { Foundational EDI Language Leaning Outcomes }}$

By the end of this module, you will:

- Gain a working understanding of common terminology utilized in equity, diversity, and inclusivity discourse.

- Reflect upon the nuanced differences between specific terms.

- Be able to identify how foundational EDI concepts may manifest in tutorial scenarios and reflect upon how one may personally navigate situations.

\section{$\underline{\text { Power, Privilege \& Positionality Learning Outcomes }}$}

By the end of this module, you will:

- Understand the terms of identity, power,

- $\quad$ privilege, intersectionality, and positionality.

- Identify your personal positionality and power dynamics in the classroom.

- Consider how your power and positionality relates to your teaching practice.

\section{Interrupting Bias Learning Outcomes}

By the end of this module, you will:

- Be able to define bias and implicit bias.

- Understand your personal biases and why biases develop.

- Be able to apply strategies for interrupting biases when they occur and maintain an inclusive learning environment. 